\title{
Nuclear Localization of Nm23-H1 in Head and Neck Squamous Cell Carcinoma Is Associated With Radiation Resistance
}

\author{
Haeng Ran Park, BS'; Se-Heon Kim, MD, PhD²; Sei Young Lee, MD, PhD³; Ji Min Sung, PhD ${ }^{4}$; Ae Ran Park, BS ${ }^{4}$; \\ Suki Kang, BS ${ }^{5}$; Baek Gil Kim, MS ${ }^{5}$; Yoon Pyo Choi, MS ${ }^{5}$; Yong-Bae Kim, MD, PhD ${ }^{6}$; and Nam Hoon Cho, MD, PhD ${ }^{1,5}$
}

BACKGROUND: Although radiation resistance is a primary issue in radiation therapy, attempts to find predictors of radiation resistance have met with little success. The authors therefore aimed to determine predictors for radiation resistance to improve the prognosis of head and neck squamous cell carcinoma (HNSCC). METHODS: HNSCC cell lines, SCC15, SCC25, and QLL1, irradiated with an acute dose of 4 grays (Gy) (RR-4), a cumulative dose of 60 Gy (RR-60), and a booster dose of 4 Gy over 60 Gy (RR-60 + 4), were used with nonirradiated cell lines. Those were used in cDNA microarray, proteomics, Western blotting, and immunofluorescence, respectively. One hundred five HNSCC tissue samples with radiation resistance were analyzed by immunohistochemistry. RESULTS: Western blot analysis of RR-60 cell lines was identical to the data of $\mathrm{Nm} 23-\mathrm{H} 1$ overexpression by cDNA array and proteomic screening. Immunofluorescence demonstrated significant nuclear translocation of Nm23-H1 in RR-4 and RR-60 cell lines, and less but still intense nuclear shuttling in RR-60 + 4. Similarly, Nm23-H1 nuclear localization was observed in $20 \%$ (21 of 105) of tissue samples. Univariate analysis demonstrated that $\mathrm{Nm} 23-\mathrm{H} 1$ nuclear localization was strongly associated with overall and recurrence-free survival. Multivariate stepwise Cox regression analysis showed that $\mathrm{Nm} 23-\mathrm{H} 1 \mathrm{nuclear}$ localization (odds ratio [OR], 7.48) and $\mathrm{N}$ stage (OR, 2.13) were associated with overall survival, and $\mathrm{Nm} 23-\mathrm{H} 1$ nuclear localization (OR, 3.02), T stage (OR, 1.43), and insufficient tumor margin (OR, 3.27) were associated with recurrence-free survival. CONCLUSIONS: Overexpression of Nm23-H1, specifically its nuclear translocation, may be a powerful predictor of radiation resistance in HNSCC. Cancer 2011;117:1864-73. (c) 2010 American Cancer Society.

KEYWORDS: Nm23-H1, nuclear localization, radiation resistance, head and neck squamous cell carcinoma, predictor.

Radiation resistance is clinically presented as locoregional failure and a serious problem for patients with advanced head and neck squamous cell carcinoma (HNSCC). Radiation resistance is responsible in part for 5-year survival rates below $50 \%$ of patients with HNSCC. ${ }^{1}$ Many attempts have been made to enhance the effectiveness of radiation therapy, including altered fractional schemes, incorporation of chemotherapy, and molecular targeted therapy. However, despite the development of antiepithelial growth factor receptor immunotherapy, small molecule kinase inhibitors, and adenoviral vectors to restore $\mathrm{p} 53$ or re-express $\mathrm{p} 16,{ }^{2}$ surgery and radiotherapy remain the preferred treatments for primary HNSCC.

Despite vigorous trials to discover biomarkers that predict radiation resistance, there have been few surrogate target markers, probably not only because the majority of candidates have depended on the exiting methods, but also because they have not been validated in clinical practice. However, biomarkers for the prediction of radiation resistance are urgently needed to improve disease-free and overall survival of patients with advanced cancers, including HNSCC. Cellular radiosensitivity is the result of a combinatorial process comprising a wide variety of signaling and effector molecules. For instance, effects of epithelial growth factor receptor function on radiosensitivity have been suggested, but the role of epithelial growth factor receptor in radiosensitivity remains controversial. ${ }^{3-5}$ In addition, Bcl-2 overexpression and p53 mutation were also associated with radiation resistance in HNSCC. ${ }^{6} \mathrm{PI} 3 \mathrm{~K}$ activation, as assessed by Akt phosphorylation,

Corresponding author: Nam Hoon Cho, MD, PhD, Department of Pathology, Yonsei University College of Medicine, 134 Shinchon-dong, Seodaemoon-gu, Seoul, Republic of Korea; Fax: (011) 82-2-362-0860; cho1988@yuhs.ac

${ }^{1}$ Brain Korea 21 Project for Medical Science, Yonsei University College of Medicine, Seoul, Republic of Korea; ${ }^{2}$ Department of Otorhinolaryngology, Yonsei University College of Medicine, Seoul, Republic of Korea; ${ }^{3}$ Department of Otolaryngology-Head and Neck Surgery, Chung-Ang University College of Medicine, Seoul, Republic of Korea; ${ }^{4}$ Department of Research Affairs, Yonsei University College of Medicine, Seoul, Republic of Korea; ${ }^{5}$ Department of Pathology, Yonsei University College of Medicine, Seoul, Republic of Korea; ${ }^{6}$ Department of Radiation Oncology, Yonsei University College of Medicine, Seoul, Republic of Korea

DOI: 10.1002/cncr.25760, Received: June 23, 2010; Revised: September 20, 2010; Accepted: October 1, 2010, Published online November 18, 2010 in Wiley Online Library (wileyonlinelibrary.com) 
or overexpression of hypoxia-inducible factors (eg, HIF1a and carbonic anhydrase 9) may predict radiation resistance in tumors showing locoregional failure. ${ }^{7,8}$ Excision repair cross-complementation group 1 has also been demonstrated to predict poor survival, ${ }^{9}$ and hedgehog activation has been reported to contribute to chemoradiation resistance in patients with esophageal cancer with radiation resistance. ${ }^{10}$ We focused on Nm23-H1 as a biological predictor of radiation resistance based on both cDNA array and proteomics in the present study. Nm23-H1 is a ubiquitously distributed nuclear diphosphate kinase that catalyzes the phosphorylation of nucleoside diphosphates. ${ }^{11}$ During tumor metastasis, Nm23-H1 may inhibit motility in response to serum and platelet-derived growth factors, ${ }^{12}$ reduce ERK activation in response to signaling, ${ }^{13,14}$ and disassemble the cytoskeleton. ${ }^{15,16}$ In the present study, we established radiation-resistant cell lines with cumulative irradiation dosages identical to the amounts administered in radiotherapy for patients with HNSCC, and retrospectively analyzed tissue samples with radiation resistance from HNSCC patients. In addition, the biological properties of $\mathrm{Nm} 23-\mathrm{H} 1$ were assessed to characterize radiation resistance in the event of Nm23-H1 nuclear localization.

\section{MATERIALS AND METHODS}

\section{Generation of Radiation-Resistant Cell Lines}

HNSCC cell lines, SCC15, SCC25, and QLL1, were cultured in minimum essential media (GIBCO-BRL, Gaithersburg, $\mathrm{Md}$ ) supplemented with $10 \%$ fetal bovine serum (FBS; GIBCO-BRL), 1\% penicillin, and 1\% streptomycin. Renal cell carcinoma cell line, caki-1, used for the acute 4 gray (Gy) dose irradiation (RR-4) test, was maintained in RPMI1640 (GIBCO-BRL) with 10\% FBS and $1 \%$ antibiotics. All cell lines were maintained at $37^{\circ} \mathrm{C}$ in a humidified atmosphere of $5 \% \mathrm{CO}_{2}$.

Cells initially treated with 2 Gy radiation were grown to $80 \%$ confluence after seeding into another flask, and aliquots accumulated a dosage of 60 Gy after 30 repetitions. Those cells were characterized as radiation resistant to the cumulative dose of 60 Gy (RR-60), with subsequent radiation of $4 \mathrm{~Gy}(\mathrm{RR}-60+4)$.

\section{cDNA Array}

All procedures from RNA extraction to cDNA microarray data analysis were done as previously described. ${ }^{17}$

The only difference between our study and previous studies is that nonirradiated cell lines RNA were labeled with cy3.

\section{Proteomics \\ Protein extraction}

Both RR-60 and nonirradiated cells were directly homogenized with a motor-driven homogenizer (PowerGen125, Fisher Scientific, Pittsburgh, Pa) in 2-dimensional (2D) gel electrophoresis lysis solution composed of $7 \mathrm{M}$ urea, $2 \mathrm{M}$ thiourea, 4\% (wt/vol) 3-([3-cholamidopropyl] dimethylammonio)-1-propanesulfonate, 1\% (wt/ vol) dithiothreitol, 2\% (vol/vol) Pharmalyte, and $1 \mathrm{mM}$ benzamidine. Proteins were extracted by vortexing for 1 hour and centrifugation at $15,000 \times g$ for $1 \mathrm{hr}$ at $15^{\circ} \mathrm{C}$. Insoluble materials were discarded, and the soluble fractions were saved for 2D gel electrophoresis. Protein concentration was measured by Bradford assay.

\section{2-DE and image analysis}

Briefly, $200 \mu \mathrm{g}$ of protein extract was separated by isoelectric focusing using an immobilized $\mathrm{pH}$ gradient strip with a nonlinear $\mathrm{pH}$ gradient of 4 to 10 (Genomine, Kyungbuk, Korea) for the first dimension, and then sodium dodecyl sulfate-polyacrylamide gel electrophoresis (SDS-PAGE; $26 \times 20 \mathrm{~cm}$ format) for the second dimension by molecular weight. Proteins were detected by alkaline silver staining.

Quantitative analysis of digitized images was carried out using PDQuest software (version 7.0, BioRad, Hercules, Calif) according to the manufacturer's protocols. Quantity of each spot was normalized by total valid spot intensity.

\section{Protein profile analysis and identification by mass spectrometry}

Clustering of samples and profiling of the protein expression were performed as described previously. ${ }^{18}$ For protein identification, a matrix-assisted laser desorption ionization time of flight (MALDI-TOF) mass spectrometer was used as previously described. ${ }^{18}$ The obtained amino acid sequence was identified by a homology search in a BLAST search using the ExPASy Molecular Biology Server (www.expasy.ch).

\section{Western Blot Analysis}

To clarify the nuclear shuttling of Nm23-H1, NE-PER Nuclear and Cytoplasm Extraction Reagents (Pierce Biotechnology, Rockford, Ill) were used to separate cytoplasmic and nuclear extracts. Equal amounts of cell extracts were subjected to SDS-PAGE and transferred to a nitrocellulose membrane (Bio-Rad). Anti-Nm23-H1 antibody (1:500 dilution, Abcam, Cambridge, UK) and 
antiaurora kinase A (AURKA) antibody (1:500 dilution, Abcam) were used as primary antibodies, and antiglyceraldehyde-3-phosphate dehydrogenase (1:500 dilution, GAPDH; Santa Cruz Biotechnology, Santa Cruz, CA) was used as the internal control. Next, membranes were washed $3 \times$ with phosphate-buffered saline (PBS)-Tween, and incubated for an additional 1 hour with horseradish peroxidase-linked secondary antibody diluted in PBSTween with 5\% skimmed milk. Donkey polyclonal antibodies against Nm23-H1 and AURKA, and rabbit polyclonal antibodies against GAPDH (1:10,000 dilution, Zymed Laboratories, San Francisco, Calif) were used as secondary antibodies.

\section{Immunofluorescence}

SCC15, SCC25, QLL1, and caki-1 cells seeded on slides were fixed with precooled methanol at $-20^{\circ} \mathrm{C}$ for 10 minutes. Cells were incubated with anti-Nm23-H1 (dilution, 1:100 in PBS-Tween) and then incubated with Alexa Fluor 594 secondary antibody (dilution, 1:250; Molecular Probes, Eugene, Ore and Carlsbad, Calif). The slides were washed $3 \times$ with PBS and mounted with ProLong Gold Antifade Reagent (Molecular Probes, P36930, Carlsbad, Calif). Cells were observed by fluorescent inverted microscopy (Olympus, Tokyo, Japan, IX71 equipped with DP71 camera). Scattergrams were used for quantification of nuclear fractionated frequencies of Nm23-H1. We analyzed all images using TissueQuest software (TissueGnostics, Vienna, Austria) to measure the total cell number from the DAPI images. Cells were plotted according to their Alexa Fluor 594 intensity versus their DAPI intensity on the scattergrams. We isolated nonspecifically stained structures and large objects from the image.

\section{Patients and Clinical Findings}

We found a total of 105 patients who were eligible for this study from patient records of the Yonsei University Health System. All patients had HNSCC (primary tumor staging: T0, 9.5\%; T1, 33.3\%; T2, 25.7\%; T3, 20.0\%; T4, 11.4\%; and regional lymph node staging: N0, $61.0 \%$; $2,31.4 \%$ ), and they had been treated with postoperative 3-dimensional (3D) conformal radiotherapy for the past 10 years (mean age \pm standard deviation, $58.2 \pm$ 11.7 years; male, $\mathrm{n}=81[77.1 \%]$ ). Mean overall survival time was 696.3 months (median, 565.5 months). Mean recurrence-free survival was 569.1 months (median, 426.0 months). All patients provided written informed consent, and the study was approved by the Research Ethics Board of Yonsei University Health System.
All patients underwent computed tomography (CT) simulation (Picker PQ5000 CT scanner, Phillips Medical Systems, Andover, Mass) with $5 \mathrm{~mm}$ thick slice cuts. Intravenous contrast agents were used in all patients. Structures were manually contoured onto the CT scan slices following the recommendations of the International Commission on Radiation Units and Measurements Report 50.

All patients received $3 \mathrm{D}$ conformal radiotherapy with single daily fractions of 2.0 Gy, 5 days per week. The planned total dose was 60 to 70.0 Gy over 6 to 7 weeks. The radiation treatment areas included the gross primary tumor as well as the regional neck nodes. Radiotherapy was performed with $4 \mathrm{MV}$ photon beams from a linear accelerator (CLINAC 600C, Varian Medical Systems, Palo Alto, Calif). Field reduction at 44 or 45 Gy was completed to exclude the spinal cord from the large fields. The remaining dose was delivered to smaller lateral or oblique fields, including only the primary tumor and clinically or radiographically detectable nodes with a margin of $1 \mathrm{~cm}$. Electron beams of suitable energy were used to boost the primary tumor site as well as grossly involved lymph nodes.

\section{Immunohistochemistry}

The $4 \mu \mathrm{m}$ tissue sections were placed on silane-coated slides, deparaffinized, immersed in PBS containing $0.3 \%$ ( $\mathrm{vol} / \mathrm{vol}$ ) hydrogen peroxide, and then processed in a microwave oven (in $10 \mathrm{mM}$ sodium citrate buffer, $\mathrm{pH}$ 6.5, for 15 minutes at $700 \mathrm{~W})$. After blocking with $1 \%(\mathrm{wt} / \mathrm{vol})$ bovine serum albumin in PBS containing $0.05 \%$ (vol/vol) Tween20 for 30 minutes, the sections were incubated with biotinlabeled rabbit anti-Nm23-H1 polyclonal antibodies (dilution, 1:2000; Abcam) and with biotin-labeled rabbit antiAURKA polyclonal antibodies (dilution, 1:400; Abcam) at $4^{\circ} \mathrm{C}$ for 16 hours, respectively. Biotinylation of the antibodies was performed with an antibody biotin-labeling kit (Envision, Dako, Glustrup, Denmark). Streptavidin-conjugated peroxidase was used as a secondary antibody $(1: 10,000)$. Normal goat serum and subtype-matched normal mouse immunoglobulin $\mathrm{G}$ were used as negative controls. The final reaction product was observed by adding $0.03 \%(\mathrm{wt} / \mathrm{vol})$ of $3,3^{\prime}$-diaminobenzidine tetrachloride for 5 to 20 minutes. Carcinomas were semiquantified using a 2-tier system ( 0 , negative staining; 1 , weakly focal positive $<50 \%$; 2 , strong positive $>50 \%$ ) which included immunolocalization of cytoplasm and/or nucleoplasm.

\section{Statistical Analysis}

Patients were categorized according to Nm23-H1 expression and localization. Time to recurrence was measured 


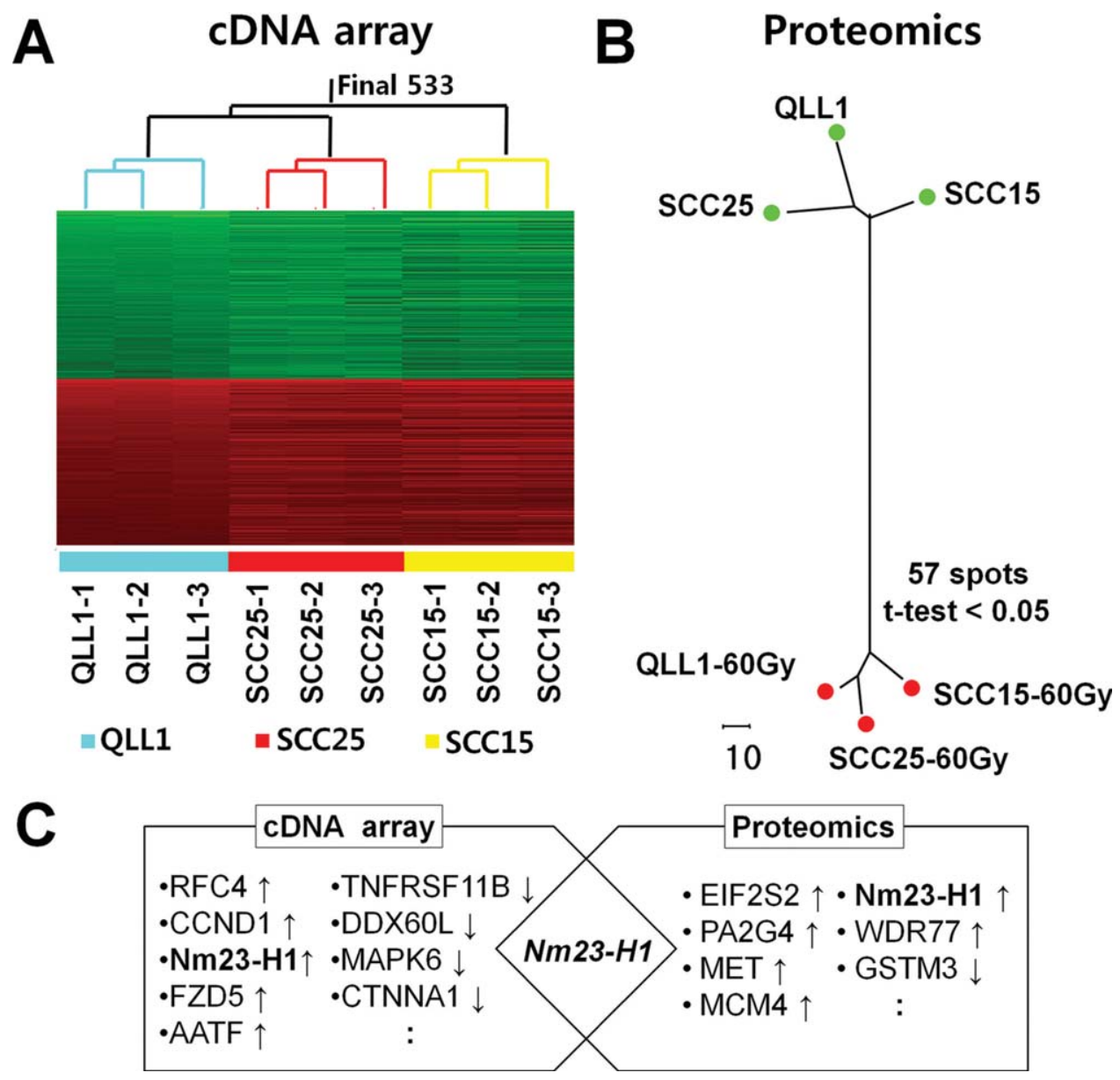

Figure 1. Screening of radiation resistance predictors by cDNA array and proteomics using 3 different head and neck squamous cell carcinoma cell lines, SCC15, SCC25, and QLL1, is shown. (A) Hierarchical clustering of CDNA array is shown. The clustering of SCC15 differed from those of SCC25 and QLL1. (B) Distance map tree represents relative distances between nonirradiated cells and RR-60 cells from a total of 57 spots $(P<.05)$. (C) Overexpression of $\mathrm{Nm} 23-\mathrm{H} 1$ is observed in both cDNA array and proteomics results.

from the date of diagnosis to the date of the first local or distant metastasis or to the last follow-up. Patients who died before disease recurrence were considered censored at the date of death. Patients who experienced local recurrence as the first recurrence were considered censored at the date of local recurrence. Times to local and distant recurrence were estimated by Kaplan-Meier analysis, and comparisons between groups were made with log-rank statistics. Cox proportional hazards models were used to determine the association between expression and nuclear localization of $\mathrm{Nm} 23 \mathrm{H} 1$ and the risk of radiation resistance as assessed by local recurrence. Each model contained terms for Nm23-H1 cytoplasmic expression, Nm23-H1 nuclear localization, age at diagnosis, tumor size, tumor type, histological grade, $\mathrm{T}$ stage, $\mathrm{N}$ stage, $\mathrm{M}$ stage, and incomplete margin. $P<.05$ was considered significant.
Statistical analyses were performed using SAS software (SAS, Cary, NC).

\section{RESULTS}

\section{Screening of Radiation Resistance-Related Genes by cDNA Array and Proteomics}

A total of 533 genes were differentially expressed in the cDNA array; 265 genes were up-regulated, and 268 genes were down-regulated in RR-60 cell lines compared with nonirradiated cells. One-way hierarchical clustering showed both up-regulated and down-regulated genes (Fig. 1A).

Fifty-seven proteins $(P<.05)$ were screened by MALDI-TOF. In analysis of the distance map tree, the distance between nonirradiated and irradiated cell lines of RR-60 (SCC15, SCC25, and QLL1) was significantly great (Fig. 1B). 

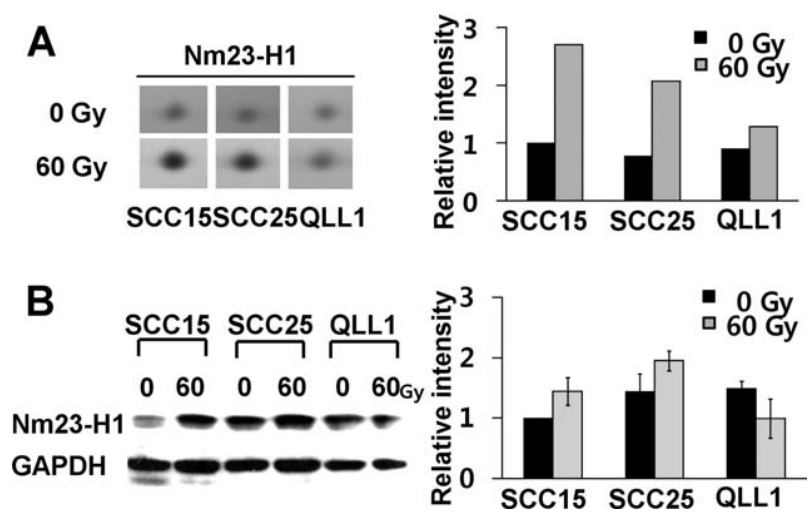

Figure 2. Validation of $\mathrm{Nm} 23-\mathrm{H} 1$ is shown. (A) Proteomics spots were identified as $\mathrm{Nm} 23-\mathrm{H} 1$. Irradiated $\mathrm{SCC} 15$ and SCC25 cell lines showed increased spot density. (B) In Western blotting, SCC15 and SCC25 cells showed high levels of $\mathrm{Nm} 23-\mathrm{H} 1$ expression in RR-60 cells, whereas the expression was low in QLL1 cells after glyceraldehyde-3-phosphate dehydrogenase (GAPDH) normalization.

In cDNA array data, DNA repair gene (RFC4), cell cycle-related gene (CCND1), or signal transduction related genes (FZD5, CTNNA1) were significantly altered with $>2$-fold expression. In addition, cell death or senescence related genes (ie, AATF, TNFRSF11B, DDX6OL, or $M A P K O)$ were significantly related. In proteomics data, most proteins related to cell cycle-related proliferation (EIF2S2, PA2G4, MET, MCM4) or DNA replication (WDR77) were up-regulated, whereas GSTM3 was down-regulated. Notably, Nm23-H1 was the only overlapping gene product that was detected in both cDNA array and proteomics (Fig. 1C).

\section{Validation of Nm23-H1 Detected by cDNA Array and Proteomics}

Both cDNA array and proteomics results showed Nm23$\mathrm{H} 1$ as the strongest predictor of radiation resistance. The Nm23-H1 spot significantly increased in RR-60 SCC15 and SCC25, but not in QLL1. Relative intensities represent the spot densities on the 2D gel. Nearly a 2-fold higher density was observed in RR-60 SCC15 and SCC25 (Fig. 2A). The immunoblotting results revealed that $\mathrm{Nm} 23-\mathrm{H} 1$ was similarly overexpressed as in the proteomics results but down-regulated in QLL1 cells (Fig. 2B).

\section{In Vitro Nuclear Translocation of Nm23-H1 by Indirect Immunofluorescence}

Immunofluorescence demonstrated that $\mathrm{Nm} 23-\mathrm{H} 1$ is generally localized in the cytoplasm in nonirradiated HNSCC cell lines. However, HNSCC cells that received a 4 Gy dose of fractionated radiation (RR-4) demonstrated nuclear localization of Nm23-H1. Nm23-H1 was rarely observed in the nuclei of HNSCC and caki-1 cells that were not irradiated. Caki-1 cells showed nuclear localization of Nm23-H1 in dot patterns after a 4 Gy dose of irradiation. Both RR-4 and RR-60 + 4 demonstrated fewer nuclear signals than RR-60, but were intensively stained (Fig. 3A). Nuclear fluorescent signals were quantified by scattergram. The proportion of $\mathrm{Nm} 23-\mathrm{H} 1$ nuclear fluorescent signals was increased in RR-60 when compared with nonirradiated cell lines (Fig. 3B). After nuclei/ cytoplasm fractionation, $\mathrm{Nm} 23-\mathrm{H} 1$ signal was elevated after a single dose of 4 Gy irradiation in nonirradiated cells. However, RR-60 cells after a 4 Gy booster dose of irradiation did not induce overexpression of $\mathrm{Nm} 23-\mathrm{H} 1$. AURKA expression showed a similar pattern with $\mathrm{Nm} 23$ H1. Nm23-H1 expression level in RR-60 of QLL1 was the same with Western blotting, as shown in Figure 2B. For this reason, the relationship between AURKA and Nm23-H1 in RR-60 cells of QLL1 was quite different from other cell lines. The relative intensity of the right panel represents the quantity of $\mathrm{Nm} 23-\mathrm{H} 1$ and AURKA in nucleus after GAPDH normalization (Fig. 3C).

\section{Immunohistochemistry of Nm23-H1 and AURKA in Clinical Samples With Radiation Resistance}

The most common finding of Nm23-H1 in HNSCC cells was intense staining, especially of tumor nests, in contrast to no Nm23-H1 staining in normal squamous epithelium (Fig. 4A). After the analysis of 105 tissue samples, only cytoplasmic Nm23-H1 expression was detected in $94(89.52 \%)$ samples. Additional nuclear expression was detected in $21(20 \%)$ samples, with 5 samples showing predominantly nuclear localization. $\mathrm{Nm} 23-\mathrm{H} 1$ distribution was accentuated along the tumor margin (Fig. 4B). Diffusely infiltrative cancer cells accompanied by a desmoplastic stromal reaction showed remarkably strong nuclear staining compared with relatively weak staining in the epicenters of tumor nests (Fig. 4C). A patchlike distribution of Nm23-H1 was apparent along the invasive front of the tumor (Fig. 4D). Nm23-H1 was diffusely localized within the nuclei of certain radiation-resistant tissues (Fig. 4E) in addition to a typical cytoplasmic expression (Fig. 4F). AURKA showed nuclear expression in all cancer cells, more intensely in invasive cells, in particular (Fig. 4G). All with Nm23-H1 nuclear expression demonstrated AURKA nuclear coexpression. 
A
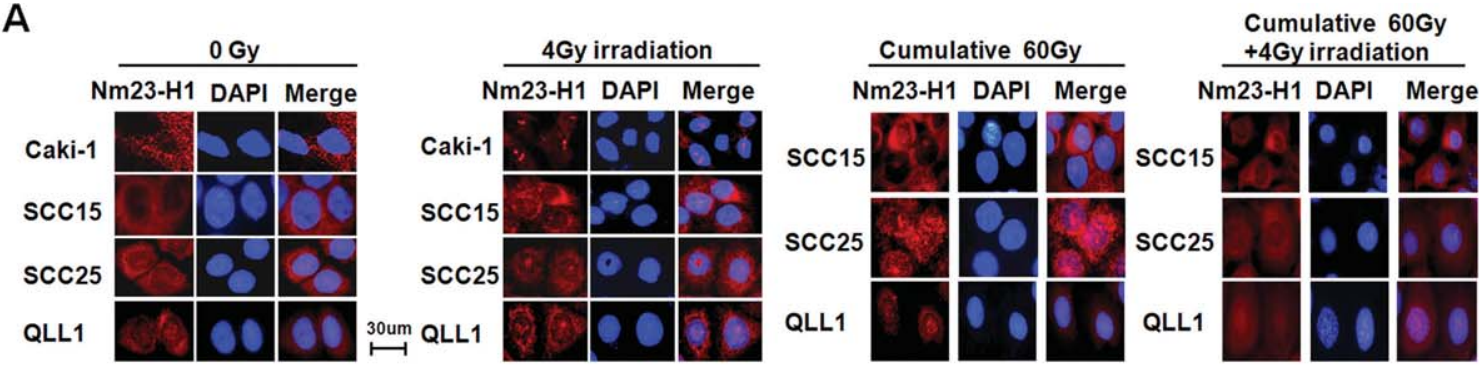

B
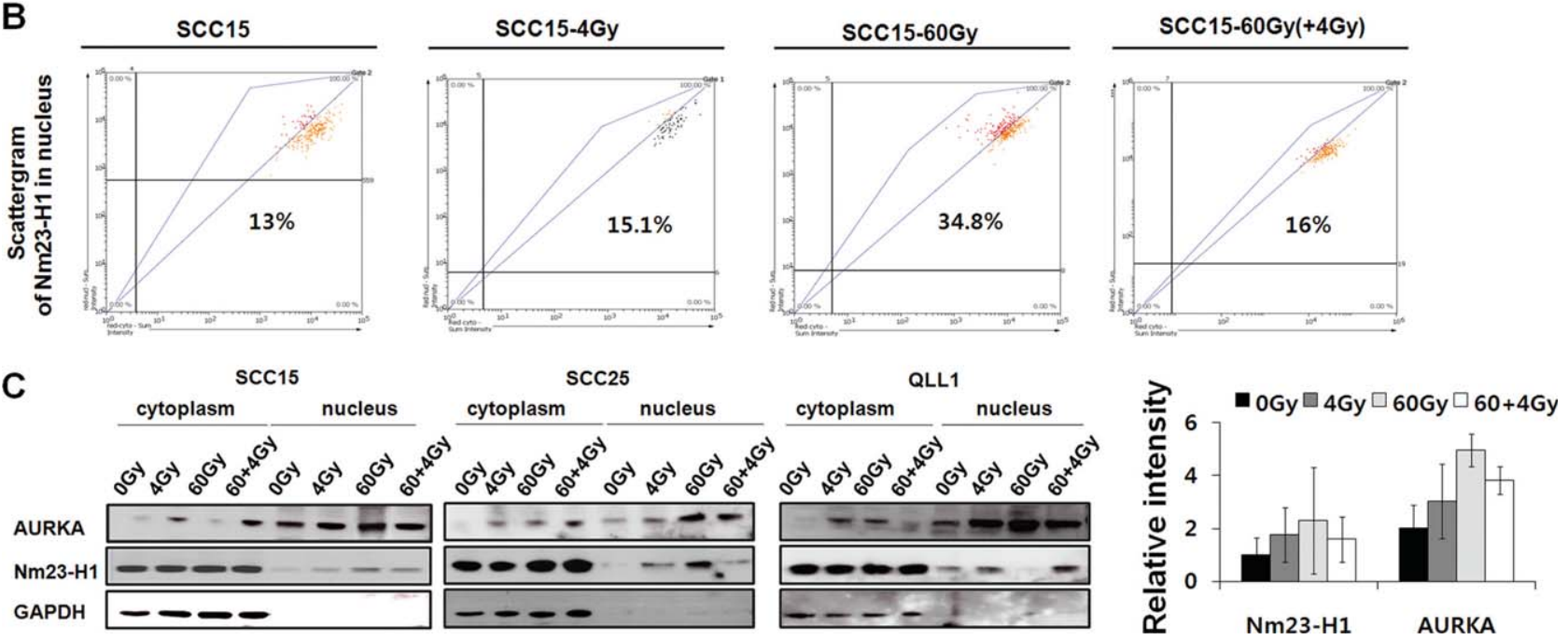

Figure 3. Nm23-H1 nuclear translocation in radiation-treated cell lines is shown. Nm23-H1 translocated to the nucleus is shown in head and neck cancer cell lines, SCC15, SCC25, and QLL1, with a 4 Gy (human median lethal dose) radiation treatment in nonirradiated cells. (A) Each panel indicates the localization and intensity of $\mathrm{Nm} 23-\mathrm{H} 1$. The first panel shows nonirradiated cells. Cells in the next panel were treated with a single 4 Gy dose of radiation. The third panel displays RR-60 cell lines, and the last panel indicates a radiation-resistant cell line (treated with a total dose of $60 \mathrm{~Gy}$ ) after a booster dose of $4 \mathrm{~Gy}$. Notice the nuclear speckled pattern in the caki-1 cell line after a $4 \mathrm{~Gy}$ dose of irradiation. (B) Quantitative analysis of immunofluorescence staining of Nm23-H1 is shown. Each scattergram represents the nucleoplasm intensity in SCC15 cell line in a radiation dose-dependent manner. (C) The expression quantity of $\mathrm{Nm} 23-\mathrm{H} 1$ and aurora kinase A (AURKA) in the nucleoplasm was estimated by Western blotting, and the highest expression was observed in RR-60 cells. The right panel represents the relative intensity of $\mathrm{Nm} 23-\mathrm{H} 1$ and AURKA after glyceraldehyde-3-phosphate dehydrogenase (GAPDH) normalization.

\section{Association of Nm23-H1 Expression With Overall or Recurrence-Free Survival}

Kaplan-Meier analysis demonstrated a significant association between cytoplasmic/nuclear expression of Nm23-H1 and overall/recurrence-free survival (Fig. 5). Analysis of overall and recurrence-free survival using the Cox proportional hazard model is displayed in Tables 1 and 2, respectively. Nuclear localization and cytoplasmic expression of $\mathrm{Nm} 23-\mathrm{H} 1$ as well as cancer $\mathrm{T}$ stage were strongly associated with overall survival in the univariate model, whereas $\mathrm{Nm} 23-\mathrm{H} 1$ nuclear localization (OR, 7.48) and $\mathrm{N}$ stage (OR, 2.13) were significant factors in the multivariate Cox hazard model (Table 1). In terms of recurrence-free survival, cytoplasmic and nuclear expressions of $\mathrm{Nm} 23-\mathrm{H} 1$ as well as insufficient tumor margins were significant in univariate analysis, and Nm23-H1 nuclear localization (OR, 2.38) and indeterminate margin (OR, 3.81) were confirmed by multivariate stepwise Cox regression analysis (Table 2).

\section{DISCUSSION}

Although many have tried to uncover biomarkers that predict radiation resistance in HNSCC, only Akt phosphorylation, overexpression of HIF 1a and carbonic anhydrase $9,,^{7,8}$ and excision repair cross-complementation group $1^{9}$ were found. Those studies were only involved in immunochemical analysis of tissues from patients with locoregional failure, which have limitation in apply for diagnosis. To find more reliable markers for radiation resistance, we established RR-60 cell lines by exposing cells to a cumulative dose of $60 \mathrm{~Gy}$ and compared them with nonirradiated cell lines using both preliminary transcriptomics and proteomics screening. The $60 \mathrm{~Gy}$ dose of irradiation 


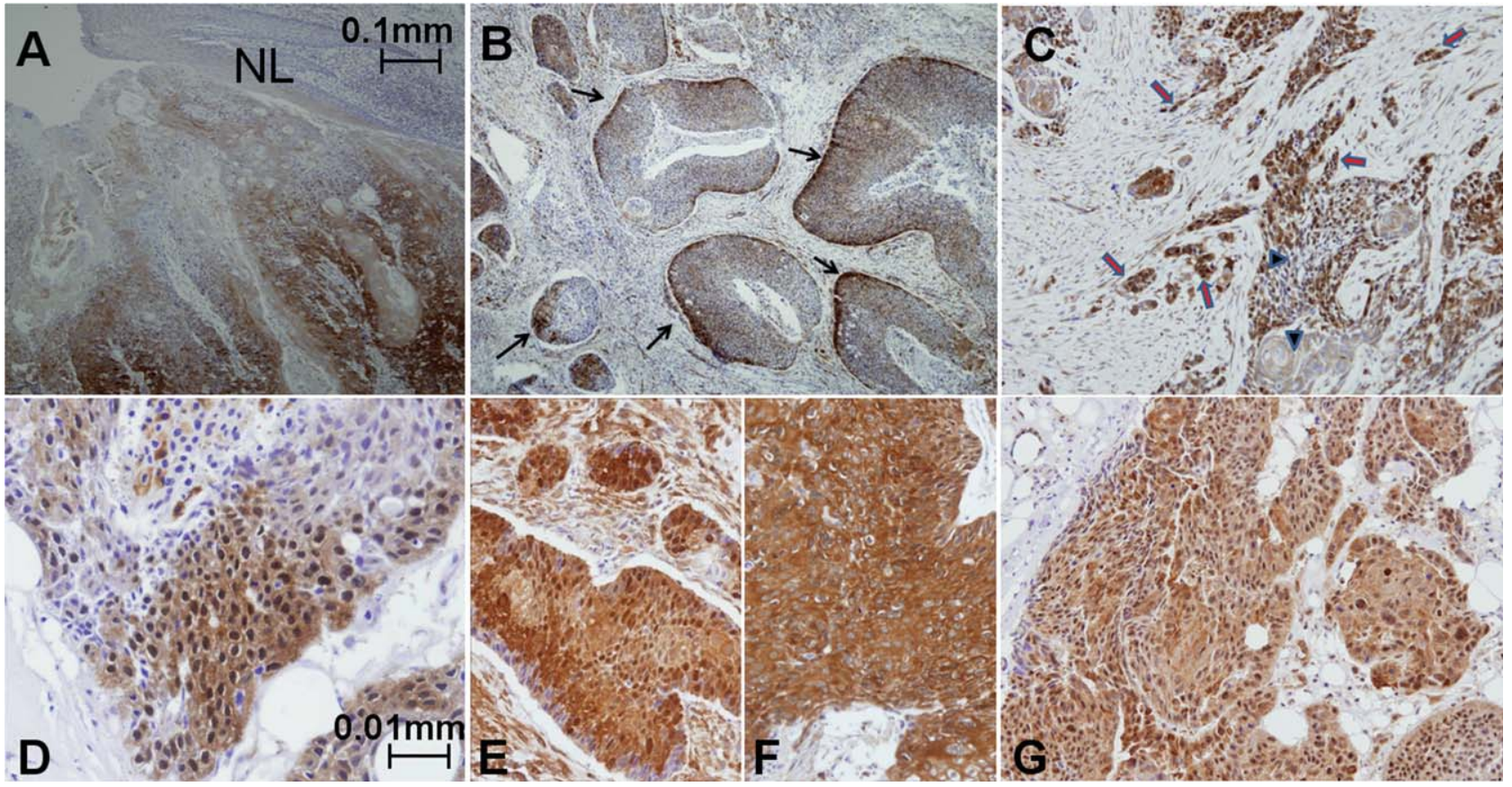

Figure 4. Immunohistochemistry of $\mathrm{Nm} 23-\mathrm{H} 1$ in head and neck squamous cell carcinoma archival tissue samples from patients who presented with locoregional failure after therapeutic radiotherapy is shown. (A) Typical expression of Nm23-H1 along the basal layers and invasive squamous cell carcinoma (SCC) are shown. Notice the negative staining in the normal squamous epithelium (NL). (B) Nm23-H1 revealed strong immunoreactivity along the margin of invasive tumor nests (arrows). (C) Remarkable nuclear staining in infiltrative tumor nests within the desmoplastic stromal reaction is shown (arrows). Note the relatively weak staining in the epicenters of SCC nests (arrowheads). (D) A patch-like distribution of $\mathrm{Nm} 23-\mathrm{H} 1$ nuclear staining in invasive fronts of the tumor is shown. (E) Diffuse nuclear localization of $\mathrm{Nm} 23-\mathrm{H} 1$ is shown. (F) Cytoplasmic staining of $\mathrm{Nm} 23-\mathrm{H} 1$ with no obvious nuclear localization identified is seen in most tumor nests. (G) Aurora kinase A showed nuclear expression in all cancer cells, more intensely in the invasive cells in particular.

A
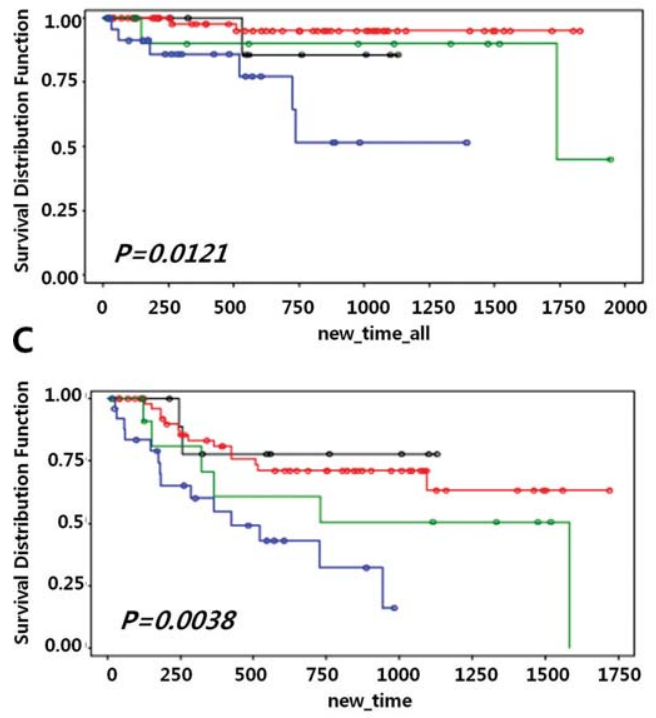

STRATA : - NM23 Cyto $=0$ Censored NM23 Cyto $=0$

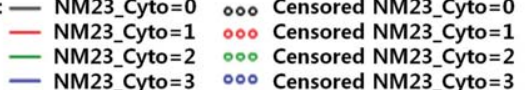

\section{B}

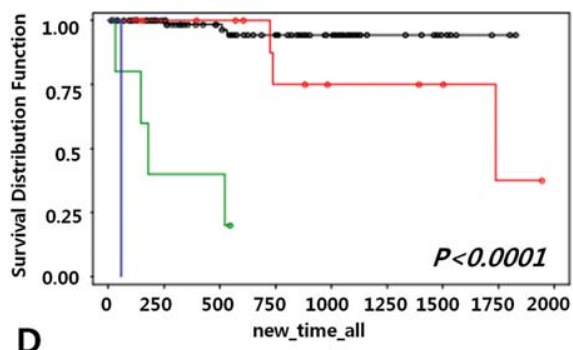

D

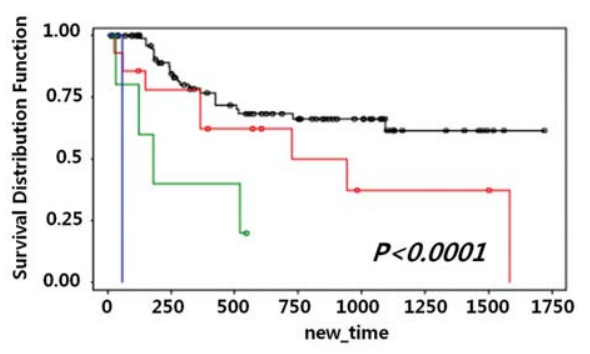
STRATA : - NM23_Nc=0 $0 \circ$ Censored NM23_Nc=0
- NM23_Nc=1 $0 \circ$ Censored NM23_Nc=1
- NM23_Nc=2
- NM23 $\mathrm{Nc}=3$

Figure 5. Kaplan-Meier analysis of $\mathrm{Nm} 23-\mathrm{H} 1$ expression and survival is shown: (A) association between $\mathrm{Nm} 23-\mathrm{H} 1 \mathrm{cytoplasmic} \mathrm{expression}$ and overall survival; (B) association between Nm23-H1 nuclear expression and overall survival; (C) association between Nm23-H1 cytoplasmic expression and recurrence-free survival; (D) association between Nm23-H1 nuclear expression and recurrence-free survival. 
Table 1. Univariate and Multivariate Overall Survival Analyses Using the Cox Proportional Hazard Model

\begin{tabular}{lllll} 
Characteristic & \multicolumn{2}{c}{ Univariate } & \multicolumn{2}{c}{ Multivariate } \\
& $P$ & HR & $P$ & HR \\
Age & .5572 & 0.99 & .1486 & 1.07 \\
Tumor margin & .0695 & 2.16 & .3265 & 3.30 \\
Tumor size & .1333 & 1.14 & .8746 & 1.07 \\
Nm23-Cyto & $.0064^{\mathrm{a}}$ & 2.54 & .2340 & 0.54 \\
Nm23-Nuc & $<.0001^{\mathrm{a}}$ & 7.54 & $<.0001^{\mathrm{a}}$ & 7.48 \\
T stage & $.0366^{\mathrm{a}}$ & 1.35 & .8138 & 1.14 \\
N stage & .1925 & 1.25 & $.0254^{\mathrm{a}}$ & 2.13
\end{tabular}

HR indicates hazard ratio; Nm23-Cyto, cytoplasmic localization of Nm23$\mathrm{H} 1$; Nm23-Nuc, nuclear localization of Nm23-H1.

${ }^{a}$ Statistically significant.

is widely used in HNSCC radiotherapy as well as investigation on tissue samples.

There were several studies using cDNA array or proteomics to discover markers for radiation resistance in HNSCC. ${ }^{19,20}$ One study found that the expression of 25 genes were altered by radiation, and 11 of them were validated in oral squamous cell carcinoma cell lines after radiation up to 8 Gy, such as ICAM2, TIMP3, PLAGL1, and FGFR3. ${ }^{20}$ In another study, 48 genes were significantly altered in myeloid cell lines (ML-1) by radiation that were primarily apoptosis-related genes such as Fas, Ciap1, Bak, and $B c l-X L .{ }^{21}$ In a study using proteomics, head and neck cancer cell lines that were exposed to a 4 Gy dose of radiation showed differential expression of proteins as follows: heat shock protein (hsp) 27, peroxiredoxin (Prx) II, and glutathione S-transferase pi (GSTP). ${ }^{22}$ Although many putative radiation resistance-related genes or proteins have been found, the demand for a reliable radiation resistance marker remained. In our study, we used both cDNA array and proteomics for screening, which may increase the reliability of radiation resistance candidates by being double checked, in RR-60 and nonirradiated cell lines. Nm23-H1 was significantly increased in RR-60 cell lines and nonirradiated cells exposed to $4 \mathrm{~Gy}$ of booster irradiation. Also, the overexpression of Nm23-H1 in nucleus was found in RR-60 cell lines. It has been reported that $\mathrm{Nm} 23-\mathrm{H} 1$ is usually located in cytoplasm, not nucleus. Therefore, we postulated that $\mathrm{Nm} 23-\mathrm{H} 1$ would have another function on radiation resistance by nuclear translocation. To determine how cells respond to radiation, especially in $\mathrm{Nm} 23-\mathrm{H} 1$ expression in nucleus, we exposed each cell line to $4 \mathrm{~Gy}$ booster radiation. The overexpression of Nm23-H1 in nucleus was observed in the nonirradiated cell lines exposed to 4 Gy of booster irradiation. However, there was no significant increase of
Table 2. Univariate and Multivariate Recurrence-Free Survival Analyses Using the Cox Proportional Hazard Model

\begin{tabular}{lllll} 
Characteristic & \multicolumn{2}{c}{ Univariate } & \multicolumn{2}{c}{ Multivariate } \\
& $P$ & HR & $P$ & HR \\
Age & .4925 & 0.99 & .4221 & 0.99 \\
Tumor margin & $.0113^{\mathrm{a}}$ & 3.17 & $.0037^{\mathrm{a}}$ & 3.81 \\
Tumor size & .8764 & 0.97 & .8320 & 0.96 \\
Nm23-Cyto & $.0009^{\mathrm{a}}$ & 1.78 & .1617 & 1.39 \\
Nm23-Nuc & $.0001^{\mathrm{a}}$ & 2.40 & $.0138^{\mathrm{a}}$ & 2.38 \\
T stage & .1315 & 1.47 & .0812 & 1.61 \\
N stage & .8953 & 0.97 & .8331 & 0.96
\end{tabular}

HR indicates hazard ratio; Nm23-Cyto, cytoplasmic localization of Nm23$\mathrm{H} 1$; Nm23-Nuc, nuclear localization of Nm23-H1.

${ }^{a}$ Statistically significant.

Nm23-H1 in nucleus of RR-60 cell lines. RR-60 cells may have already acquired $\mathrm{Nm} 23-\mathrm{H} 1$ overexpression in nucleus by repeated radiation. The assumption can be supported by the report that $\mathrm{Nm} 23-\mathrm{H} 1$ may play a role in DNA damage repair or the induction of DNA synthesis. ${ }^{23,24} \mathrm{Nm} 23-\mathrm{H} 1$ mediated DNA repair maintains genomic stability after irradiation or UV irradiation. ${ }^{23,24}$ DNA repair from irradiation targeted to double-stranded DNA of cancer cells may lead to survival of cancer.

In addition, we validated predictor $(\mathrm{Nm} 23-\mathrm{H} 1)$ expression in a large number of tissues from HNSCC patients with locoregional failure. As we mentioned above, $\mathrm{Nm} 23-\mathrm{H} 1$ is normally found in cytoplasm, and its nuclear localization has seldom been reported. However, we observed nuclear localization of Nm23-H1 in HNSCC. The most common finding of $\mathrm{Nm} 23-\mathrm{H} 1$ was cytoplasmic staining, with accentuation along the tumor margins or invasive fronts, but nuclear and cytoplasmic coexpression was also detected in $20 \%$ of a total samples. We noticed accentuation along the tumor margins or invasive fronts of $\mathrm{Nm} 23-\mathrm{H} 1$, which indicate that $\mathrm{Nm} 23-\mathrm{H} 1$ is related to with invasive potential and poor prognostic factor. In addition to distribution patterns, multivariate Cox hazard model also showed nuclear localization as a significant poor prognostic factor. Our finding is consistent with another study reporting Nm23-H1 nuclear localization as a progressive marker in breast cancer. ${ }^{25}$ Both nuclear and cytoplasmic staining were seen in $24.3 \%$ of lymph node-positive infiltrating ductal carcinoma (IDC) and $18.9 \%$ of matched lymph node metastasis cases, compared with $4.9 \%$ staining in lymph node-negative IDC. ${ }^{25}$

The functional mechanism of Nm23-H1 nuclear translocation as a poor prognostic factor has not been clarified. With specific regard to the functional mechanism, Fan 
et al mentioned that granzyme A secreted from cytotoxic $\mathrm{T}$ cells can induce the single-strand DNA nick of target cells via the nuclear translocation of Nm23-H1. ${ }^{26}$ In another report, Nm23-H1 interacts with AURKA, which might have a function in radioresistance. ${ }^{27,28}$ AURKA is a centrosome-associated kinase, and its overexpression in rodent and human cancer cells causes improper centrosome duplication, aneuploidy, and cellular transformation. ${ }^{27}$ Accumulation of $\mathrm{Nm} 23-\mathrm{H} 1$ on the centrosome coincides with the enrichment of AURKA at the centrosome and with increased AURKA activity in the beginning of mitosis through a microtubule-independent mechanism. ${ }^{28}$ On the basis of references and our results, Nm23-H1 may play a role in DNA damage repair or the induction of DNA synthesis together with AURKA. AURKA showed a coexpression pattern with Nm23-H1 in SCC15 and SCC25, but not in QLL1. AURKA showed nuclear expression in all cancer cell lines, and its expression pattern overlapped with $\mathrm{Nm} 23-\mathrm{H} 1$ in most cases. According to coexpression with AURKA in the event of nuclear translocation of Nm23$\mathrm{H} 1$, we suggest another putative pathway by which Nm23$\mathrm{H} 1$ directly interacts with AURKA in head and neck cancer undergoing radiation resistance.

$\mathrm{Nm} 23-\mathrm{H} 1$ is a reliable candidate for radiation resistance prediction in HNSCC. There has not been any report on $\mathrm{Nm} 23-\mathrm{H} 1$ overexpression in nucleus and nuclear translocation in other cancers such as thyroid cancer, ovarian cancer, T-cell lymphoma, and neuroblastomas after irradiation. ${ }^{29-32}$ Also, there are several reports that $\mathrm{Nm} 23-\mathrm{H} 1$ expression is associated with a favorable prognosis or shows a lack of association with head and neck cancer. $^{33,34}$ Thus, Nm23-H1 is thought to have tissuespecific roles with different regulatory mechanisms, and its overexpression may be specific to HNSCC.

In conclusion, nuclear translocation of $\mathrm{Nm} 23-\mathrm{H} 1$ may be a meaningful and strong predictor of radiation resistance in patients with HNSCC.

\section{CONFLICT OF INTEREST DISCLOSURES}

This research was supported by the Mid-Career Researcher Program (No. 2010-0000357; Nam Hoon Cho) and National Nuclear R\&D Program (No. 2009-0079165; Nam Hoon Cho) through a National Research Foundation grant funded by the Korea government's Ministry of Education Science and Technology.

\section{REFERENCES}

1. Hardisson D. Molecular pathogenesis of head and neck squamous cell carcinoma. Eur Arch Otorhinolaryngol. 2003; 260:502-508.
2. Le Tourneau C, Siu LL. Molecular-targeted therapies in the treatment of squamous cell carcinomas of the head and neck. Curr Opin Oncol. 2008;20:256-263.

3. Dassonville O, Formento JL, Francoual M, et al. Expression of epidermal growth factor receptor and survival in upper aerodigestive tract cancer. J Clin Oncol. 1993;11:1873-1878.

4. Wen QH, Miwa T, Yoshizaki T, Nagayama I, Furukawa M, Nishijima H. Prognostic value of EGFR and TGF-alpha in early laryngeal cancer treated with radiotherapy. Laryngoscope. 1996;106:884-888.

5. Thariat J, Yildirim G, Mason KA, Garden AS, Milas L, Ang KK. Combination of radiotherapy with EGFR antagonists for head and neck carcinoma. Int J Clin Oncol. 2007; 12:99-110.

6. Gallo O, Chiarelli I, Boddi V, Bocciolini C, Bruschini L, Porfirio B. Cumulative prognostic value of p53 mutations and bcl-2 protein expression in head-and-neck cancer treated by radiotherapy. Int J Cancer. 1999;84:573-579.

7. Gupta AK, McKenna WG, Weber CN, et al. Local recurrence in head and neck cancer: relationship to radiation resistance and signal transduction. Clin Cancer Res. 2002;8 :885-892.

8. Koukourakis MI, Giatromanolaki A, Danielidis V, Sivridis E. Hypoxia inducible factor (HIflalpha and HIF2alpha) and carbonic anhydrase 9 (CA9) expression and response of head-neck cancer to hypofractionated and accelerated radiotherapy. Int J Radiat Biol. 2008;84:47-52.

9. Kim MK, Cho KJ, Kwon GY, et al. ERCC1 predicting chemoradiation resistance and poor outcome in oesophageal cancer. Eur J Cancer. 2008;44:54-60.

10. Sims-Mourtada J, Izzo JG, Apisarnthanarax S, et al. Hedgehog: an attribute to tumor regrowth after chemoradiotherapy and a target to improve radiation response. Clin Cancer Res. 2006;12:6565-6572.

11. Lascu L. The nucleoside diphosphate kinases 1973-2000. J Bioenerg Biomembr. 2000;32:213-214.

12. Kantor JD, McCormick B, Steeg PS, Zetter BR. Inhibition of cell motility after nm23 transfection of human and murine tumor cells. Cancer Res. 1993;53:1971-1973.

13. Hartsough MT, Morrison DK, Salerno M, et al. Nm23-H1 metastasis suppressor phosphorylation of kinase suppressor of Ras via a histidine protein kinase pathway. J Biol Chem. 2002;277:32389-32399.

14. Salerno M, Palmieri D, Bouadis A, Halverson D, Steeg PS. $\mathrm{Nm} 23-\mathrm{H} 1$ metastasis suppressor expression level influences the binding properties, stability, and function of the kinase suppressor of Ras1 (KSR1) Erk scaffold in breast carcinoma cells. Mol Cell Biol. 2005;25:1379-1388.

15. Eckes B, Dogic D, Colucci-Guyon E, et al. Impaired mechanical stability, migration and contractile capacity in vimentin-deficient fibroblasts. J Cell Sci. 1998;111:18971907.

16. Otero AS. Copurification of vimentin, energy metabolism enzymes, and a MER5 homolog with nucleoside diphosphate kinase. Identification of tissue-specific interactions. J Biol Chem. 1997;272:14690-14694.

17. Ki DH, Jeung HC, Park $\mathrm{CH}$, et al. Whole genome analysis for liver metastasis gene signatures in colorectal cancer. Int $\mathrm{J}$ Cancer. 2007;121:2005-2012.

18. Kim DS, Choi YP, Kang S, et al. Panel of candidate biomarkers for renal cell carcinoma. J Proteome Res. 2010;9: 3710-3719. 
19. Frederick BA, Helfrich BA, Coldren CD, et al. Epithelial to mesenchymal transition predicts gefitinib resistance in cell lines of head and neck squamous cell carcinoma and nonsmall cell lung carcinoma. Mol Cancer Ther. 2007;6:16831691.

20. Ishigami T, Uzawa K, Higo M, et al. Genes and molecular pathways related to radioresistance of oral squamous cell carcinoma cells. Int J Cancer. 2007;120:2262-2270.

21. Amundson SA, Bittner M, Chen Y, Trent J, Meltzer P, Fornace AJ Jr. Fluorescent cDNA microarray hybridization reveals complexity and heterogeneity of cellular genotoxic stress responses. Oncogene. 1999;18:3666-3672.

22. Lee YS, Chang HW, Jeong JE, Lee SW, Kim SY. Proteomic analysis of 2 head and neck cancer cell lines presenting different radiation sensitivity. Acta Otolaryngol. 2008;128:8692.

23. Kaetzel DM, Zhang Q, Yang M, McCorkle JR, Ma D, Craven RJ. Potential roles of $3^{\prime}-5^{\prime}$ exonuclease activity of NM23-H1 in DNA repair and malignant progression. $J$ Bioenerg Biomembr. 2006;38:163-167.

24. Yang M, Jarrett SG, Craven R, Kaetzel DM. YNK1, the yeast homolog of human metastasis suppressor NM23, is required for repair of UV radiation- and etoposide-induced DNA damage. Mutat Res. 2009;660:74-78.

25. Ismail NI, Kaur G, Hashim H, Hassan MS. Nuclear localization and intensity of staining of $\mathrm{nm} 23$ protein is useful marker for breast cancer progression. Cancer Cell Int. 2008;8:6.
26. Fan Z, Beresford PJ, Oh DY, Zhang D, Lieberman J. Tumor suppressor NM23-H1 is a granzyme A-activated DNase during CTL-mediated apoptosis, and the nucleosome assembly protein SET is its inhibitor. Cell. 2003;112:659-672.

27. Guan Z, Wang XR, Zhu XF, et al. Aurora-A, a negative prognostic marker, increases migration and decreases radiosensitivity in cancer cells. Cancer Res. 2007;67:10436-10444.

28. Du J, Hannon GJ. The centrosomal kinase Aurora-A/ STK15 interacts with a putative tumor suppressor NM23H1. Nucleic Acids Res. 2002;30:5465-5475.

29. An HJ, Kim DS, Park YK, et al. Comparative proteomics of ovarian epithelial tumors. J Proteome Res. 2006;5:10821090.

30. Niitsu N, Nakamine $H$, Okamoto $M$, et al. Expression of $\mathrm{nm} 23-\mathrm{H} 1$ is associated with poor prognosis in peripheral Tcell lymphoma. Br J Haematol. 2003;123:621-630.

31. Takeda O, Handa M, Uehara $T$, et al. An increased NM23H1 copy number may be a poor prognostic factor independent of $\mathrm{LOH}$ on $1 \mathrm{p}$ in neuroblastomas. Br J Cancer. 1996;74:1620-1626.

32. Zou M, Shi Y, al-Sedairy S, Farid NR. High levels of $\mathrm{Nm} 23$ gene expression in advanced stage of thyroid carcinomas. Br J Cancer. 1993;68:385-388.

33. Gunduz M, Ayhan A, Gullu I, et al. nm23 Protein expression in larynx cancer and the relationship with metastasis. Eur J Cancer. 1997;33:2338-2341.

34. Wang YF, Chow KC, Chang SY, et al. Prognostic significance of $\mathrm{nm} 23-\mathrm{H} 1$ expression in oral squamous cell carcinoma. Br J Cancer. 2004;90:2186-2193. 